\title{
Erratum to: The electric quadrupole moment of molecular hydrogen ions and their potential for a molecular ion clock
}

\author{
D. Bakalov $\cdot$ S. Schiller
}

Published online: 30 July 2014

(c) Springer-Verlag Berlin Heidelberg 2014

\section{Erratum to: Appl. Phys. B (2014) 114:213-230 \\ DOI 10.1007/s00340-013-5703-z}

In the paper by D. Bakalov and S. Schiller, published in Appl. Phys. B 114, 213-230 (2014); doi:10.1007/s00340013-5703-z, a mismatch of the normalization conventions for the cyclic components of irreducible tensors of rank two has lead to errors in some of the equations with impact on the numerical results about the electric quadrupole shift of the spectral lines of the molecular hydrogen ions. To make these equations consistent and the numerical results correct, the following changes are required:

1. Equation (17) on page 215 becomes

$$
\begin{aligned}
& \left\langle\lambda v^{\prime} L^{\prime} M^{\prime}\left|\Delta H_{Q}\right| \lambda v L M\right\rangle \\
& =-\frac{1}{2}\left(\sum_{q=-2}^{2} \tilde{Q}^{q}\left(\mathbf{R}_{C}\right) C_{L M, 2 q}^{L^{\prime} M^{\prime}}\right) \\
& \quad \times\left(2 L^{\prime}+1\right)^{-1 / 2}\left\langle\lambda v^{\prime} L^{\prime}|| \tilde{\Theta}_{C} \| \lambda v L\right\rangle
\end{aligned}
$$

2. Equation (20) on page 216 becomes

The online version of the original article can be found under doi:10.1007/s00340-013-5703-z.

\section{Bakalov}

Institute for Nuclear Research and Nuclear Energy, Tsarigradsko

chaussée 72, 1784 Sofia, Bulgaria

\section{S. Schiller $(\square)$}

Institut für Experimentalphysik, Heinrich-Heine-Universität

Düsseldorf, 40225 Düsseldorf, Germany

e-mail: step.schiller@uni-duesseldorf.de

$$
\begin{aligned}
V^{\mathrm{Q}}(v, L) & =\sqrt{\frac{3}{2}} E_{14}(v, L) Q\left(\mathbf{R}_{C}\right) \cdot(\mathbf{L} \otimes \mathbf{L})^{(2)} \\
& =\frac{3}{2} E_{14} \sum_{q} \tilde{Q}^{q} \tilde{\mathcal{L}}_{q}
\end{aligned}
$$

3. In the right-hand sides of Eqs. (36), (37), (39), (40) and (42), the coefficient $E_{14}$ is replaced by $\left(\frac{3}{2} E_{14}\right)$. In particular, Eq. (40) becomes

$$
\begin{aligned}
\Delta E_{\mathrm{Q}, \mathrm{diag}}^{\nu L F S J_{z}}= & \left(\frac{3}{2}\right)^{\frac{3}{2}} \\
& \times E(v, L) Q_{z z}\left\langle\nu L F S J J_{z}\left|L_{z}^{2}-\frac{1}{3} \mathbf{L}^{2}\right| v L F S J J_{z}\right\rangle
\end{aligned}
$$

4. In Sect. 4.5, the examples are intended to illustrate uniquely the issue of accuracy of the diagonal approximation and are essentially numerical considerations; the chosen values of the cyclic components are not necessarily physical (the stated case where $\tilde{Q}^{1}$, $\tilde{Q}^{-1}$ are nonzero and equal real numbers contradicts the symmetry properties of the tensor $\tilde{Q}$ ) or realistic.

5. In line 6 of Sect. 4.5, insert a comma before "for a gradient".

6. In Sect. 4.5, rescale the assumed values of all components of the gradient tensor, $\tilde{Q}^{q}$, by $2 / 3$.

7. In the examples mentioned in the sentence "All Zeeman states..." of Sect. 4.5, the value of $\tilde{Q}^{0}$ is the same as in the earlier examples of the section, i.e., now $67 \mathrm{MV} \mathrm{m}^{-2}$.

8. In line 9 from the bottom of page 221 , replace “... a value $Q_{z z}=0.1 \mathrm{GV} \mathrm{m}^{-2}$ " by “... a value $Q_{z z}=67$ $\mathrm{MV} \mathrm{m}^{-2}$ ".

9. In the caption to Table 4 on page 223 , replace “... gradient has its only non-vanishing component... 
$Q=\tilde{Q}^{0}=Q_{z z}=0.1 \mathrm{GV} \mathrm{m}^{-2}$ ” by “... gradient tensor has its only non-vanishing cyclic component ... $\tilde{Q}^{0}=Q_{z z}=67 \mathrm{MV} \mathrm{m}^{-2} »$.
10. In the caption to Table 5 on page 224 , replace " $Q_{z z}=10^{8} \mathrm{~V} / \mathrm{m}^{2}$ " by " $Q_{z z}=67 \mathrm{MV} / \mathrm{m}^{2}$ ". 